\title{
Predicting Growth and Trends of COVID-19 by Implementing Machine Learning Algorithms
}

\author{
Daniel Mago Vistro ${ }^{1, *}$ Muhammad Shoaib Farooq ${ }^{2}$, Attique Ur Rehman ${ }^{2}$, \\ Muhammad Omer Aftab ${ }^{2}$
}

\author{
${ }^{1}$ School of Computing, Asia Pacific University, Kuala lumpur, Malaysia. \\ ${ }^{2}$ School of System and Technology, University of Management and Technology, Pakistan. \\ *Corresponding author. Email: Daniel.mago@staffemail.apu.edu.my
}

\begin{abstract}
Artificial Intelligence has absolutely revolutionized the world in which we live, and with the passing of time it advances exponentially. The applications of AI are tremendous like healthcare and medical solutions, disease diagnostics, agriculture, developing security infrastructures, Autonomous vehicles, intelligent systems, industrial manufacturing, robotics and so much more. COVID19 is a deadly virus that started from china in 2019 and started to spread rapidly and within time spread throughout various countries of world and in 2020 the world went to a huge pandemic and many lives were lost due to this deadly virus causing to a major health hazard. Moreover, in 2021 many countries experience other new forms of the Covid19 that are faster to spread and more deadly. The spread and growth need to me monitored and evaluated to control the spread. The paper states the proposed methodology to evaluate insights of the growth rate or number of cases along with the death rate of COVID19 to getter better visualization to impose lockdown and area evacuation for population safety. We have applying popular Machine Learning algorithms for the forecast of COVID19 including Naive Bayes, Bayes Net, Decision Tree, Random Forest, Logistic Regression. Moreover, the technique will help to evaluate the trend to get better insights for behaviour analysis of COVID19. This study would aid policymakers in taking the required steps in advance, such as preparing isolation wards, ensuring the supply of drugs and paramedical staff, deciding partial or complete lockdown strategies, recruiting volunteers, and developing economic strategies. Out of all techniques, Random Forest algorithm outstands others with the highest accuracy of $87.28 \%$ with precision and recall of $89 \%$ and $85 \%$ respectively.
\end{abstract}

Keywords: Artificial Intelligence (AI), AI Solution, Covid-19, Deep Learning (DL), Health Care Solution, Machine Learning (ML).

\section{INTRODUCTION}

Artificial Intelligence has absolutely revolutionized the world in which we live, and with the passing of time it advances exponentially. Many AI technologies are being developed, providing creative and useful solutions to difficult problems for the improvement of humanity. For improved decision making, conventional and manual systems are now being converted to autonomous systems. The applications of AI are tremendous like healthcare and medical solutions, disease diagnostics, agriculture, developing security infrastructures, Autonomous vehicles, intelligent systems, industrial manufacturing, robotics and so much more [1][2] [40, 41] [48-52]. Such creative applications are increasingly relevant in society and are becoming more widespread. Popular areas where
AI is found to be productive and reliable include continuously and rapidly analysing vast volumes of data and information or where it is possible to simplify mundane operating activities [3]. Covid-19 is a deadly virus that started from china in 2019 and started to spread rapidly and within time spread throughout various countries of world and in 2020 the world went to a huge pandemic. From 2019 till Feb 2020 there are 106,555,206 confirmed cases of Covid-19 worldwide while the number of deaths reached to number of 2,333,446 as reported by WHO [4] [42-45]. The top 5 countries having the most number of cases are USA, India, Brazil, Russia, United Kingdom, [5]. Figure1 states the demographics of top 10 most Covid-19 cases countries worldwide and figure 2 depicts the daily cases and death rate of COVID19 from Jan 2020 to Mar 2021 [6]. 
COVID-19's biological structure consists of a positive-oriented single-stranded form of RNA and the disease is difficult to manage because of its mutating function. Medical professionals are conducting extensive studies globally to determine an appropriate treatment for the disease. [7]. There are several forms of coronavirus, and these viruses are commonly seen in animals. In humans, bats, pigs, cats, dogs, rodents, and poultry, COVID-19 has been found. Pain in the throat, headache, fever, runny nose, and cough are symptoms of COVID19.

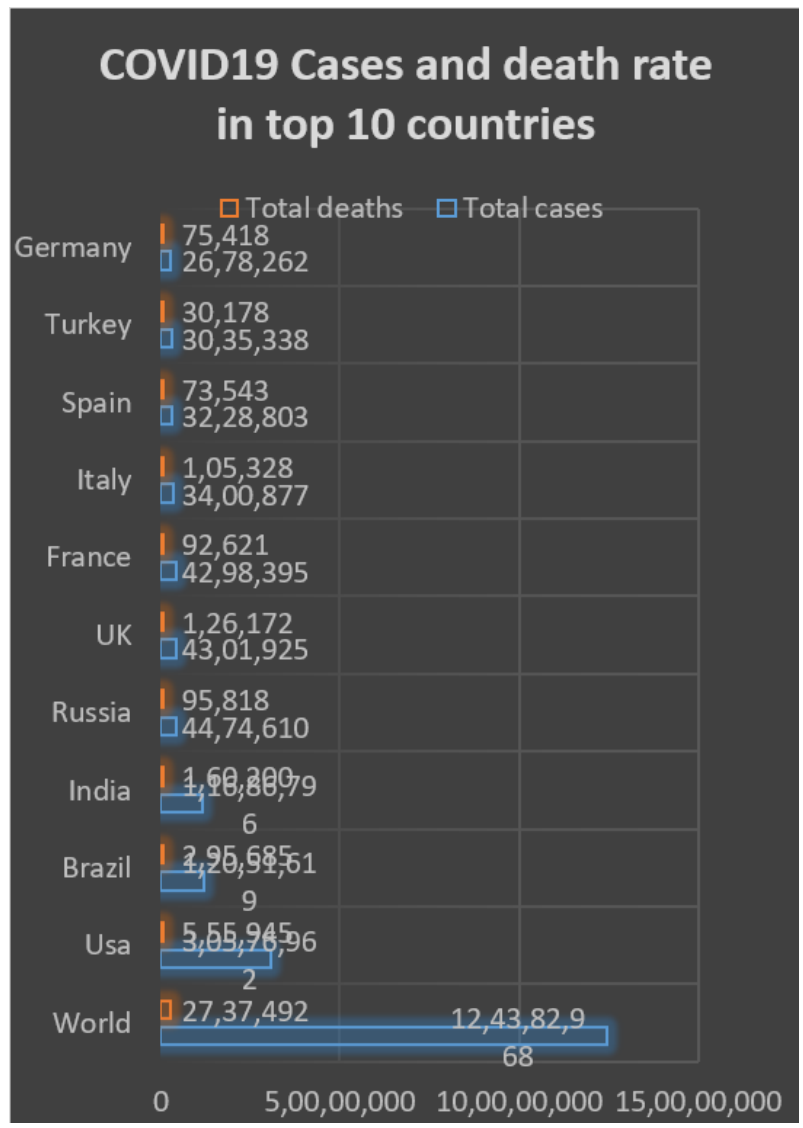

Figure 1 COVID case statistics of top 10 countries 2021[6].

The virus can cause individuals with weakened immune systems to die [8]. Accurate classifications of the population dependent on classified COVID-19 vulnerability will greatly benefit from tailored preventive strategies. A new discovery that more and more young adults suffer from serious COVID-19 symptoms contradicts the earlier observation that older individuals have a greater risk for COVID-19, suggesting an immediate need for a systematic risk assessment based on personalized genetic and physiological characteristics [9].

COVID-19 is primarily transferred from person to person by direct touch. COVID-19 is transmitted by breath touch, hand contact, or mucous contact in healthy persons. Artificial intelligence (AI) has been extensively used to speed up biomedical research in recent years.
People infected with COVID-19 will develop pneumonia if the virus spreads to the lungs. Several deep learning studies have detected the disease using chest X-ray image images. Deep learning approaches have been used in a variety of applications, including pattern recognition, data classification, and image segmentation [10].

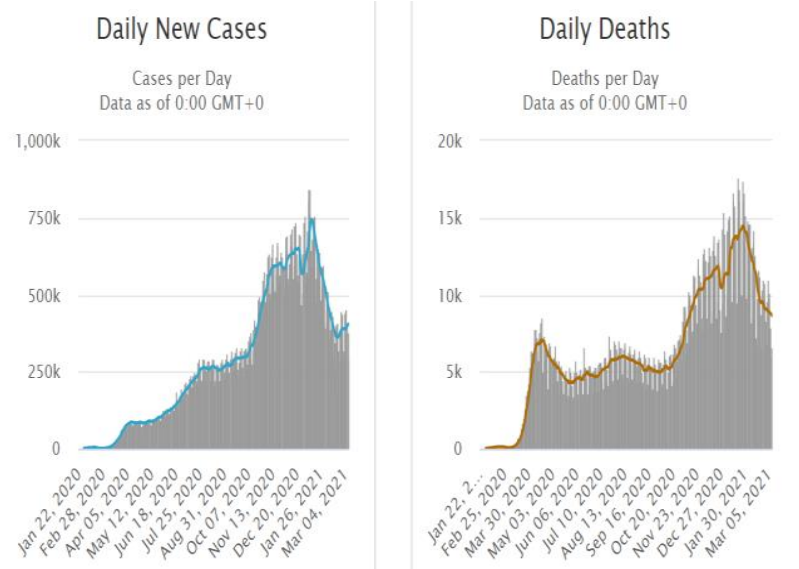

Figure 2 COVID cases and death rate from Jan 2020 to Mar 2021[6].

The deep neural network, which has several layers of linear and non-linear operations, is one of the most popular DL models (DNN). DNN is a deep neural network (DNN) that extends the traditional neural network by adding several hidden layers, allowing the model to learn more complex input data representations. Recurrent neural networks (RNNs), which can map sequential input data to their output, are the deepest architectures of DL algorithms. The nodes in each RNN layer are bound to one another, unlike typical DNNs. RNNs benefit from this self-connection because it helps them remember things over time as they are given a series of data [11][12]. Convolutional neural networks, on the other hand, are a form of DNN influenced by a human visual cortex (CNN). CNN employs three types of layers: convolution, pooling, and fully linked layers. Convolution and pooling layers are used in the lower levels. Convolution layers generate a series of linear activations followed by non-linear functions. In practice, the convolution layers employ filters to reduce the sophistication of the input data. The pooling layers are then used to down sample the filtered data. The pooling layers help to reduce the size of the activation maps by transferring them into a smaller matrix [13], [14], [15]. To train a model to produce specific classifications for data with unknown labels, a dataset of images with defined labels is used. The algorithm is trained by feeding it known data and modifying model parameters iteratively to produce correct labels for taxa that are known to be represented in the picture. Protocols for preparing a precise CNN [16]. Algorithms are increasingly evolving, and transfer learning, a tool for updating and optimizing pre-trained CNNs for new applications, has lowered the requirement for training 
images needed, allowing for comparatively low training costs. Weak deployment of results [17], [18] [46, 47]. Although healthcare AI conversion is imminent and undeniable, there are a few challenges that need to be addressed. The fact that an AI algorithm needs to have a proper input a huge amount of properly labelled data for proper output is one of the biggest challenges, and that is difficult to obtain in the current healthcare system [19]. In other words, the fact that $\mathrm{AI}$ algorithms require more and more computing power requires stronger hardware for larger data sets. Another concern that needs to be tackled is that due to convergence and overfitting, AI models may be unreliable, something that will probably have to be solved in the years that follow [20]. And we must mention the privacy of information, one of the biggest concerns in today's world. AI data sets provide a large volume of patient-related personal knowledge. Data is money, and a lot of data is a lot of money, a fact that we are reminded of every time a data breach happens. We cannot expect full AI integration into the healthcare system without a solution to that problem [21].

The paper outlines a model for evaluating the growth rate or number of cases, as well as the mortality rate in COVID19, in order to obtain a clearer picture of how to impose lockdown and area evacuation for population protection. For the COVID19 forecast, we used Naïve Bayes, Bayes Net, Decision Tree, Random Forest, and Logistic Regression, among other common Machine Learning algorithms. Furthermore, the approach will aid in evaluating the pattern in order to gain deeper information for COVID19 behaviour analysis.

The paper is organized into sections, first section is the introduction section where we have stated information, facts and figures and relevant data for readers. Second section is the related work where we have listed state-of-the-art work relevant to the proposed methodology. Next is the problem statement which states the problem and objective of the methodology. Forth section is the proposed methodology which states the data and technique used, architecture and software process flow. Fifth section is the simulation and results here the carried out experiment and simulation with results are mention. Last is the conclusion where we have listed our findings and future work.

\section{RELATED WORK}

Three different calculations for Contaminated I ( $\mathrm{t}$ ), Deaths $\mathrm{D}(\mathrm{t})$, and Recovery $\mathrm{R}(\mathrm{t})$ have been considered, including the currently positive population $\mathrm{P}(\mathrm{t})$. The model simulations demonstrate the SARS-CoV-2 pandemic simulations for assessing the impact of potential policies such as social isolation and lockout. The findings of our model, which were obtained by combining the SARIIqSq model with data on the coronavirus outbreak in India, indicates that social distancing is important, central, and efficient. No work on death rate or recovery rate has been done only predicts COVID19 outcast on limited areas [23]. COVID-19 epidemiological data is used in the logistic model to meet the epidemic trend boundary, and then feed the cap value into the FbProphet model, a machine-learning-based time series simulation model that extracts the epidemic curve and forecasts the epidemic trend. The worldwide epidemic will peak in late October, with an estimated 14.12 million cumulatively infected persons, according to statistical projections. Piecewise prediction with a variant of Logistic model deserves further study. Research is limited to only times series also lack the application on real time data [24]. An AI-based approach to screening prospective integrative medicine therapies will be incredibly helpful in reducing the risk of COVID19. Integrative medicine and artificial intelligence (AI) are two new approaches that could aid in the development of novel solutions and assist in the battle against this deadly pandemic. There is no evidence of their practical application only qualitative reasoning is stated [25].

A newly designed algorithm was which was based on a well-known Trust-region-reflective (TRR) While our proposed disease model only uses a few analytical parameters, uncertainty analysis will reveal a lot about the quantitative interaction between model responses and model input mechanisms. Just a few countries were studied, and predictions for COVID19 outcasts were made, but no study was performed to forecast death rates [26]. Two approaches for calculating the number of individuals infected with the current coronavirus have been proposed (COVID-19). With figures and confidence intervals of 95 percent, the total number of predicted cases and deaths worldwide is $12.7 \times 106$ and $5.27 \times 105$, respectively, as of 06-06-2020. This study is limited for academic and research purposes only, and the predictions for the future are based on the assumption that the current restrictive conditions would continue [27]. The ARIMA and LSSVM data modelling techniques are used in this study to predict one month out of sample data points, resulting in an estimate of a rise in possible COVID-19 reported events. To render non-stationary data stationary, a rational differential transformation is applied. The ARIMA model parameters $\mathrm{p}$ and $\mathrm{q}$ are measured by regenerating ACF and PACF stationary data plots obtained via the differentiation method. The study data is limited to one month of COVID19 cases and only for two countries so the model is limited to fewer resources [28]. Adaptive online Incremental Learning methodology based on ANN, where model parameters are constantly modified to respond intelligently to new data sets. We suggest and model a risk-dependent population compartmentalization (PC) controlled natural immunization mechanism in which the population is divided into low risk (LR) and high risk (HR) compartments. For efficient regression analysis, the ANN employs a non-linear activation mechanism. Whose layers are concealed is determined by the FNN 
function. The paper lacks in the practical implementation the study is limited to academic and research perspective [29].

The progression of the disease is assessed and predicted using updated mathematical analytics using an enhanced ML-based model. Predictions using the Robust Weibull method by region, as well as the error relationship between the Robust Weibull and the Baseline Gaussian Model. It is therefore forecasted when the total volume will reach 97 percent of the expected anticipated events. The paper lacks in the practical implementation the study is limited to academic and research perspective [30]. The autonomous evaluation of COVID-19, which is focused on deep learning and provides simple and precise remedies, has aided in the diagnosis of this condition. Emerging methods that combine integrative medicine and artificial intelligence (AI) could produce unique solutions and help in the battle against COVID19, but there is no evidence of their practical application [31]. On this front, we tried a few different assembly processes, but none of them resulted in any more changes. CT-BERT proved to be extremely successful. The results emphasize the relevance of domain and task-related pre-training. Although one model outperforms the other two, it is also possible that they each have unique strengths that lead to better outcomes when combined. Finally, but not least, can SVM, neural network, and BERT-based models be combined to increase resiliency [32]. Transfer learning pre-trained models to construct a multi-label classifier that can characterize conspiracy theory details. As a new video classification feature, we use the percentage of comments on misinformation on each video. In addition, we perform a Bayesian analysis to determine the function's value. After this, we show that using first ever hundred comments as tf - idf features increases the video classifier's precision. Misinformation identification on social media is still a work in progress, and more analysis is needed to explain how the COVID19 miss infodemic spread and deter future outbreaks [33].

In this section various relative techniques for the prediction and detection of COVID19 are stated. It has been witnessed that for the prediction of the outcast or trends or growth of COVID19 a majority of ML based techniques have been used while for the detection of COVID19 most Deep learning techniques have been used so we can say this problem comes up with diverse solutions and techniques. The proposed work is novel as in other techniques 1, 2 algorithms are applied and results are compared, or the dataset used is limited to for one country or fewer or either the dataset is composed of a fewer no of instances while the proposed methodology is built to predict the death rate and growth rate using latest time series dataset with over $22 \mathrm{k}$ instances from around the world. Moreover, 5 classification machine learning approaches have been used for the better prediction of COVID19 and results are compared which algorithm well suits the problem. And provide more accurate results.

\section{PROPOSED MATERIAL AND METHODS}

The methodology will help to gain insights about the growth of COVID19 in accordance with the new cases and death rate that will help to impose various precautionary measures to apply smart lockdown strategies to increase paramedical staff in a certain area where there is an expected risk of increasing COVID19 cases or for the behavioural analysis of the disease. In the proposed methodology the Covid19 prediction is being carried out in 2 ways, first we are predicting the global confirmed cases second, we are predicting the global death rate of Covid19. Figure 2 shows the datasets being used in state-of-the-art methodologies. Weka and Jupiter Notebook are used in the proposed methodology due to their efficient and outstanding results using less time. For this purpose, the "Global time series datasets are used". The dataset is divided into 2 portions the global confirmed cases and death rate of Covid19. The total number of instances are 22852 while the number of attributes is 10 that represents country, country code, continent, population, indicator, weekly count, date, rate14-day, cumulative and source.

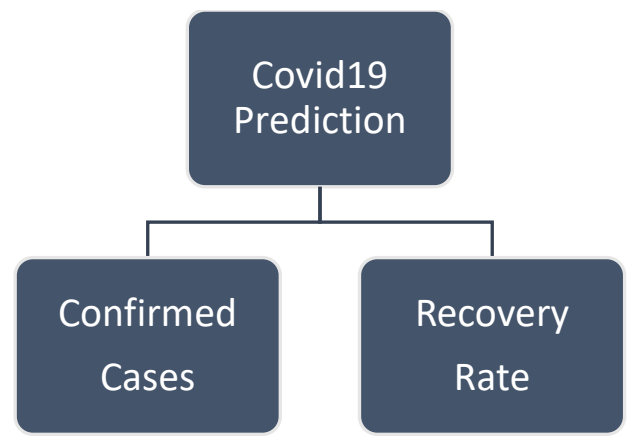

Figure 3 Types of Covid19 Datasets used.

In the proposed methodology various machine learning classification techniques are applied that are Naïve Bayes, Bayes Net, Decision Tree, Random Forest, and Logistic Regression. The dataset consists of 10 attributes from which the 'indicator' attribute is the class attribute consisting of two classes 'cases' and 'deaths'. Figure 4 shows the proposed architecture the first layer is the data acquisition layer here in which the data is gathered in raw form which needs to be improved and cleansed. For data improvement next is the data preprocessing layer where some pre-processing techniques are applied like finding missing values, normalization, and noise removal. The pre-processing operations plays a very important task in improvement of data as the data must be into a uniform format for better results of the data. Next comes the main part the application layer that comprises of two layers prediction and performance evaluation layer. First step is the training of data her 
implementation of classification techniques, as mentioned we have applied 5 different algorithms using Jupiter notebook and Weka. For the training phase the pre-processed cleaned data is loaded into the model and the training process will begin. Once the training process is completed next comes the performance evaluation phase which is very important as it helps to evaluate the model's performance.

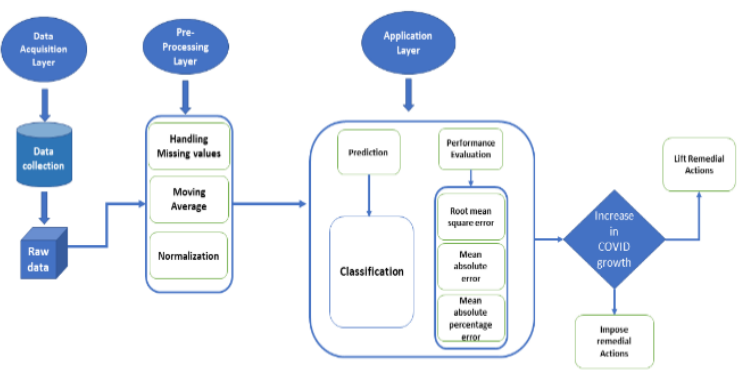

Figure 4 Purposed System Architecture.

Root mean square mean absolute error and mean absolute error percentage are the performance evaluation parameters that are used on the bases of these parameters the accuracy of the model is determined. On the bases of the final output in accordance with the growth prediction of COVID19 the decision can be made to lift the imposed remedial actions or there is further needed to apply remedial actions. Figure 5 depicts the detail insights of the application layer. The application layer consists of classification algorithms used for the prediction of COVID19 on the time series dataset. For classification 5 algorithms have been used are Naïve Bayes, Bayes Net, Decision Tree, Random Forest and Logistic Regression. Here the dataset will be fed into each algorithm and the evaluation of performance will be carried out using Root mean square mean absolute error and mean absolute error percentage. These parameters will help to compare the performance of each algorithm at the end to select which algorithm suits the problem and presents with most accurate results.

Equation 1 states the mathematical equation of Naïve Bayes algorithm here $\mathrm{A}$ and $\mathrm{B}$ are the events where $\mathrm{P}$ $(A \mid B)$ is the probability of $A$ given $B$ is true. $P(B \mid A)$ is the probability of $B$ given $A$ is true. $P(A)$ and $P(B)$ are the independent probabilities of event $\mathrm{A}$ and $\mathrm{B}$. Where equation 2 shows the classifier where $\mathrm{y}^{\wedge}$ is the predicted value. Equation 3 shows the mathematical model of random forest algorithm this method calculates the Gini of each branch on a node based on the class and likelihood, deciding which branch is more likely to occur. The relative frequency of the class you are looking at in the dataset is represented by pi, and the number of classes is represented by c. Equation 4 shows the entropy of random forest algorithm.

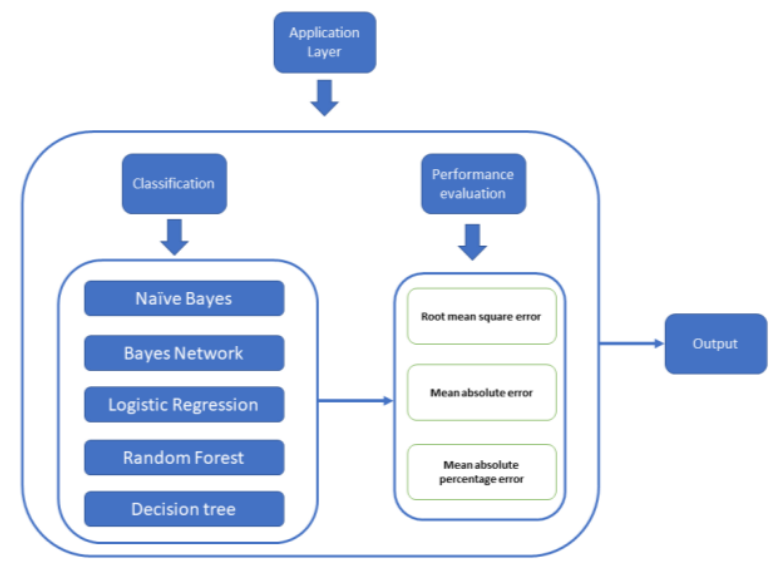

Figure 5 Types of Covid19 Datasets used.

Entropy is used to decide if the node can branch depending on the likelihood of a given result. Because of the logarithmic function used to calculate it, it is more mathematically intensive than the Gini index. Equation 5 and 6 show how to calculate the info (D). In terms of the amount of detail needed to better define the tree, this attribute provides us with the splitting attribute. Knowledge benefit reduces the amount of data used to assign data points into partitions while still reflecting the least amount of randomness or "impurity" in these partitions. Info (D) is the average amount of data required to determine the class/category of a data point in D. Since most information is encoded in bits, a log function to base 2 is used. Equation 7 and 8 shows the way to calculate gain ratio of input attributes. The knowledge benefit metric favours experiments with many results. As a result, it chooses attributes of a wide number of properties. Gain ratio is a method of addressing this problem.

$$
\begin{aligned}
& P(A \mid B)=\frac{P(B \mid A) P(A)}{P(B)} \\
& \hat{y}=\underset{y}{\operatorname{argmax}} P(y) \prod_{i=1}^{n} P\left(x_{i} \mid y\right) \\
& \text { Gini }=1-\sum_{i=1}^{C}\left(p_{i}\right)^{2} \\
& \text { Entropy }=\sum_{i=1}^{C}-p_{i} * \log _{2}\left(p_{i}\right) \\
& \operatorname{Info}_{(D)}=-\sum^{m} p_{i} \log _{2}\left(p_{i}\right), \\
& \operatorname{Info}_{A}(D)=\sum_{j=1}^{v} \frac{\left|D_{j}\right|}{|D|} \times \operatorname{Info}\left(D_{j}\right) \\
& \operatorname{Gain}(A)=\operatorname{Info}(D)-\operatorname{Info}(D) \\
& \operatorname{GainRatio}_{A}(A)=\frac{\operatorname{Gain}(A)}{\operatorname{SplitInfo}(A)} .
\end{aligned}
$$




\section{SIMULATION AND RESULTS}

The total number of instances are 22852 while the number of attributes is 10 that represents country, country code, continent, population, indicator, weekly count, date, rate-14-day, cumulative and source. Once the dataset is loaded into the model its resultant scatter plot is shown in fig.6. The scatter plot shows the correlation within the attributes of the dataset here there two sets of numerical results, one on each axis, to see if there is a connection between them. The points will fall into a line or curve if the variables are connected. The more the points embrace the axis, the better the connection.

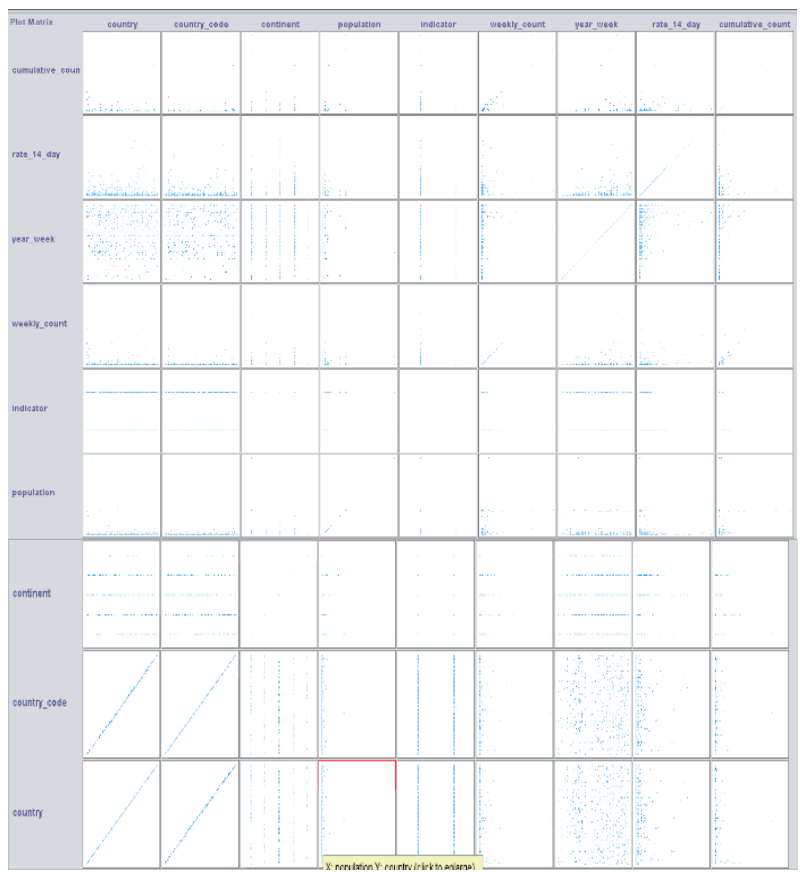

Figure 6 Scatter plot of proposed dataset.

As mentioned before the proposed methodology consist of 5 different classification algorithms Naïve Bayes, Bayes Net, Decision Tree, Random Forest, and Logistic Regression. For each algorithm, the dataset is same as the pre-processing techniques used. As mention earlier the 'indicator' attribute is the class attribute that consists of two classes 'cases' and 'deaths'. When applied logistic regression algorithm where from the total number of instances the number of correctly classified instances are 15888 while the incorrect classified instances are 6964 with the accuracy of $69.52 \%$ and with 0.71 and 0.64 of precision and recall. When applied Bayes Net algorithm where from the total no of instances the correctly classified instances are 13801 with 9051 incorrect classified instances, the accuracy was $60.39 \%$ with precision and recall of 0.73 and 0.64 , respectively. When applied Naïve Bayes algorithm where from the total no of instances the correctly classified instances are 15661 with 7191 incorrect classified instances, the accuracy was $68.53 \%$ with precision and recall of 0.68 and 0.63 , respectively. When applied RandomForest algorithm where from the total no of instances the correctly classified instances are 19946 with 2906 incorrect classified instances, the accuracy was $87.28 \%$ with precision and recall of 0.89 and 0.84 , respectively. When applied Decision Tree algorithm where from the total no of instances the correctly classified instances are 18137 with 4715 incorrect classified instances, the accuracy was $79.36 \%$ with precision and recall of 0.80 and 0.78 , respectively. The model performance of the classification algorithms is presented in the figures shown before.

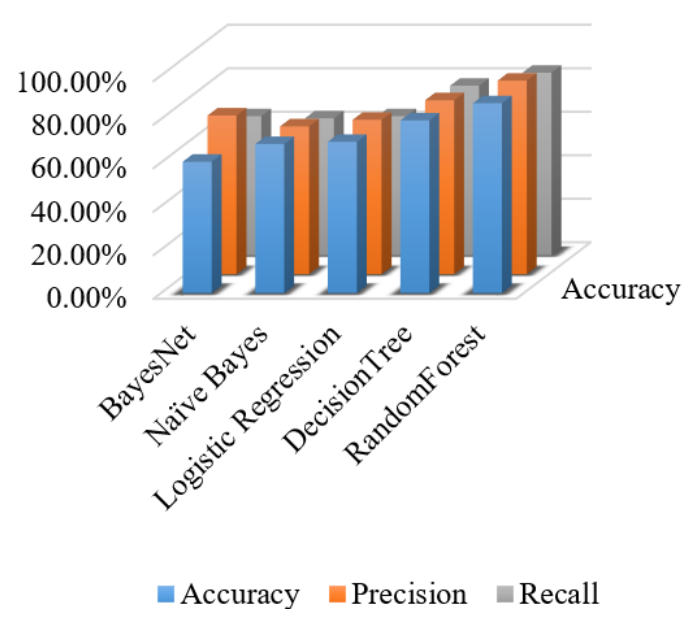

Figure 7 Performance evaluation of implemented algorithm.

After implementing all models, the performance needs to be compared. Figure 7 shows the comparison of all proposed implemented algorithms in accordance with their accuracy where out of all the algorithms the algorithm with the highest accuracy was Random Forest. Table 1 shows the comparison of the proposed work with other relative methodologies.

\section{CONCLUSION}

Covid-19 is a deadly virus that started from china in 2019 and started to spread rapidly and within time spread throughout various countries of world and in 2020 the world went to a huge pandemic. Many researchers and scientists use AI techniques as an effective weapon against Covid 19 and come up with different strategies for spread and prediction forecasting along with detection. The paper outlines a model for evaluating the growth rate or number of cases, as well as the mortality rate in COVID19, in order to obtain a clearer picture of how to impose lockdown and area evacuation for population protection. For the COVID19 forecast, we used Naïve Bayes, Bayes Net, Decision Tree, Random Forest, and Logistic Regression, among other common Machine Learning algorithms. Furthermore, the approach will aid in evaluating the pattern in order to gain deeper information for COVID19 behaviour analysis. For each algorithm, the dataset is same as the pre-processing 
techniques use. Out of all algorithms applied, Random Forest algorithm outstands others with the highest accuracy of $87.28 \%$. The methodology will help to gain insights about the growth of COVID19 in accordance with the new cases and death rate that will help to impose various precautionary measures to apply smart lockdown strategies to increase paramedical staff in a certain area where there is an expected risk of increasing COVID19 cases or for the behavioural analysis of the disease. Increasing the accuracy, efficiency and performance is a future goal and trying other techniques to enhance the results.

\section{REFERENCES}

[1] [1] Terri Park, AI Cures: data-driven clinical solutions for Covid-19. MIT News, 2020.

[2] Swami Sivasubramanian, How AI and machine learning are helping to fight COVID-19. World Economic Forum, 2021.

[3] Genevieve Smith \& Ishita Rustagi, The Problem With COVID-19 Artificial Intelligence Solutions and How to Fix Them. Standford Social Innovation Review, 2020.

[4] WHO, WHO Coronavirus Disease (COVID-19) Dashboard. World Health Organization, 2021.

[5] D. WOOD, S. ADELINE, R. TALBOT, and T. WILBURN, Coronavirus World Map: We've Now Passed The 100 Million Mark For Infections. NPR, 2021.

[6] Khan, N. S., Shahzada, A., Ata, S., Abid, A., Farooq, M. S., Mushtaq, M. T., \& Khan, I. (2014). A vision based approach for Pakistan sign language alphabets recognition.

[7] K. C. Santosh, "COVID-19 Prediction Models and Unexploited Data," J. Med. Syst., vol. 44, no. 9, pp. 1-4, 2020, doi: 10.1007/s10916-020-01645-z.

[8] K. Sarkar, S. Khajanchi, and J. J. Nieto, "Modeling and forecasting the COVID-19 pandemic in India," Chaos, Solitons and Fractals, vol. 139, p. 110049, 2020, doi: 10.1016/j.chaos.2020.110049.

[9] A. Alimadadi, S. Aryal, I. Manandhar, P. B. Munroe, B. Joe, and X. Cheng, "Artificial intelligence and machine learning to fight covid19," Physiol. Genomics, vol. 52, no. 4, pp. 200-202, 2020, doi: 10.1152/physiolgenomics.00029.2020.

[10] G. Pinter, I. Felde, A. Mosavi, P. Ghamisi, and R. Gloaguen, "COVID-19 Pandemic Prediction for Hungary; A Hybrid Machine Learning Approach," SSRN Electron. J., 2020, doi: 10.2139/ssrn.3590821

[11] Goodfellow I, Abadie JP, Mirza M, Xu B, Farley DW, Ozair S, Courville A, Bengio Y (2014)
Generative adversarial nets. In: Neural Information Processing Systems, pp 2672-2680.

[12] Raza, M. I., Zaib, Q. J., Farooq, M. S., Abid, A., \& Khan, S. A. (2012). Meticulous analysis of Semantic Data Model-An optimal approach for ERD. J. Basic. Appl. Sci. Res, 8(2), 8344-8354.

[13] Xiao C, Choi E, Sun J. Opportunities and challenges in developing deep learning models using electronic health records data: a systematic review. J Am Med Inform Assoc 2018; 25 (10): 1419-28.

[14] Jain S, Wallace BC. Attention is not explanation. In: proceedings of the NAACL-HLT 2019; 2019: 3543 56. http://arxiv.org/abs/1902.10186. 43. Devlin J, Chang M-W, Lee K, et al. Bert: pre-training of deep bidirectional transformers for language understanding. arXiv Preprint arXiv: 181004805; 2018.

[15] Peters ME, Neumann M, Iyyer M, et al. Deep contextualized word representations. arXiv Preprint arXiv: 180205365; 2019: 26 (11), 1297- 1304. https://doi.org/10.1093/jamia/ocz096

[16] Y. LeCun, Y. Bengio, G. Hinton, Deep learning. Nature 521, 436-444 (2015).

[17] J. Yosinski, J. Clune, Y. Bengio, H. Lipson, How transferable are features in deep neural networks? Adv. Neural Inf. Process. Syst. 27, 3320-3328 (2014).

[18] S. Christin, E. Hervet, N. Lecomte, Applications for deep learning in ecology. ' Methods Ecol. Evol. 10, 1632-1644 (2019).

[19] K. Cranmer, J. Brehmer, G. Louppe, The frontier of simulation-based inference. Proc. Natl. Acad. Sci. U.S.A., 10.1073/pnas.1912789117 (2020).

[20] P. L. Bartlett, P. M. Long, G. Lugosi, A. Tsigler, Benign overfitting in linear regression. Proc. Natl. Acad. Sci. U.S.A., 10.1073/pnas.1907378117 (2020).

[21] D. Bau et al., Understanding the role of individual units in a deep neural network. Proc. Natl. Acad. Sci. U.S.A., 10.1073/pnas.1907375117 (2020).

[22] K. Sarkar, S. Khajanchi, and J. J. Nieto, "Modeling and forecasting the COVID-19 pandemic in India," Chaos, Solitons and Fractals, vol. 139, p. 110049, 2020, doi: 10.1016/j.chaos.2020.110049.

[23] G. Martelloni and G. Martelloni, "Modelling the downhill of the Sars-Cov-2 in Italy and a universal forecast of the epidemic in the world," Chaos, Solitons and Fractals, vol. 139, no. March, p. 110064, 2020, doi: 10.1016/j.chaos.2020.110064. 
[24] P. Wang, X. Zheng, J. Li, and B. Zhu, "Prediction of epidemic trends in COVID-19 with logistic model and machine learning technics," Chaos, Solitons and Fractals, vol. 139, p. 110058, 2020, doi: 10.1016/j.chaos.2020.110058.

[25] A. S. Ahuja and O. Marques, "Integrative Medicine Research Artificial intelligence and COVID-19?: A multidisciplinary approach," Integr. Med. Res., vol. 9, no. 3, p. 100434, 2020, doi: 10.1016/j.imr.2020.100434.

[26] K. N. Nabi, "Forecasting COVID-19 pandemic: A data-driven analysis," Chaos, Solitons and Fractals, vol. 139, p. 110046, 2020, doi: 10.1016/j.chaos.2020.110046.

[27] Farid, S., \& Rehman, A. U. (2018). Enhancement in Quality of Services Using Integrated Services in $4 \mathrm{G}$ Cellular Network. Technical Journal, 23(03), 82-93.

[28] S. Singh, K. S. Parmar, S. J. S. Makkhan, J. Kaur, S. Peshoria, and J. Kumar, "Study of ARIMA and least square support vector machine (LS-SVM) models for the prediction of SARS-CoV-2 confirmed cases in the most affected countries," Chaos, Solitons and Fractals, vol. 139, p. 110086, 2020, doi: 10.1016/j.chaos.2020.110086.

[29] J. Farooq and M. A. Bazaz, "A novel adaptive deep learning model of Covid-19 with focus on mortality reduction strategies," Chaos, Solitons and Fractals, vol. 138, 2020, doi: 10.1016/j.chaos.2020.110148.

[30] D. P. Kavadi, R. Patan, M. Ramachandran, and A. H. Gandomi, "Partial derivative Nonlinear Global Pandemic Machine Learning prediction of COVID 19," Chaos, Solitons and Fractals, vol. 139, p. 110056, 2020, doi: 10.1016/j.chaos.2020.110056.

[31] M. Islam, F. Karray, R. Alhajj, S. Member, and J. Zeng, "A Review on Deep Learning Techniques for the Diagnosis of Novel Coronavirus ( COVID-19)," vol. 1, pp. 1-18.

[32] A. Giovanni Møller, R. van der Goot, and B. Plank, "NLP North at WNUT-2020 Task 2: Pre-training versus Ensembling for Detection of Informative COVID-19 English Tweets," pp. 331-336, 2020, doi: 10.18653/v1/2020.wnut-1.44.

[33] J. Carlos, M. Serrano, O. Papakyriakopoulos, and S. Hegelich, "NLP-based Feature Extraction for the Detection of COVID-19 Misinformation Videos on YouTube," ACL 2020 Work. NLP-COVID, no. 2018, 2020, [Online]. Available: https://openreview.net/forum?id=M4wgkxaPcyj.

[34] Vistro, D. M., Munawar, A., Iftikhar, A., Qasim, A., \& Rehman, A. U. (2020). TERTIARY CARE HOSPITAL MONITORING SYSTEM USING
WIRELESS SENSORS. Journal of Critical Reviews, 7(10), 1504-1511..

[35] H. Panwar, P. K. Gupta, M. K. Siddiqui, R. MoralesMenendez, and V. Singh, "Application of deep learning for fast detection of COVID-19 in X-Rays using nCOVnet," Chaos, Solitons and Fractals, vol. 138, p. 109944, 2020, doi: 10.1016/j.chaos.2020.109944.

[36] S. Bharati, P. Podder, and M. R. H. Mondal, "Hybrid deep learning for detecting lung diseases from X-ray images," Informatics Med. Unlocked, vol. 20, p. 100391, 2020, doi: 10.1016/j.imu.2020.100391.

[37] X. Xu et al., "A Deep Learning System to Screen Novel Coronavirus Disease 2019 Pneumonia," Engineering, vol. 6, no. 10, pp. 1122-1129, 2020, doi: 10.1016/j.eng.2020.04.010.

[38] T. Ozturk, M. Talo, E. Azra, U. Baran, O. Yildirim, and U. R. Acharya, "Automated detection of COVID-19 cases using deep neural networks with X-ray images," Comput. Biol. Med., vol. 121, no. April, p. 103792, 2020, doi: 10.1016/j.compbiomed.2020.103792.

[39] S. Hassantabar, M. Ahmadi, and A. Sharifi, "Diagnosis and detection of infected tissue of COVID-19 patients based on lung x-ray image using convolutional neural network approaches," Chaos, Solitons and Fractals, vol. 140, p. 110170, 2020, doi: 10.1016/j.chaos.2020.110170.

[40] Ngo, T.D., Bui, T.T., Pham, T.M., Thai, H.T., Nguyen, G.L. and Nguyen, T.N., 2021. Image deconvolution for optical small satellite with deep learning and real-time GPU acceleration. Journal of Real-Time Image Processing, pp.1-14.

[41] Chowdary, M.K., Nguyen, T.N. and Hemanth, D.J., 2021. Deep learning-based facial emotion recognition for human-computer interaction applications. Neural Computing and Applications, pp.1-18.

[42] Poongodi, M., Nguyen, T.N., Hamdi, M. and Cengiz, K., 2021. A Measurement Approach Using Smart-IoT Based Architecture for Detecting the COVID-19. Neural Processing Letters, pp.1-15.

[43] Subramani, P., Srinivas, K., Sujatha, R. and Parameshachari, B.D., 2021. Prediction of muscular paralysis disease based on hybrid feature extraction with machine learning technique for COVID-19 and post-COVID-19 patients. Personal and Ubiquitous Computing, pp.1-14.

[44] Prabu, S., Velan, B., Jayasudha, F.V., Visu, P. and Janarthanan, K., 2020. Mobile technologies for contact tracing and prevention of COVID-19 
positive cases: a cross-sectional study. International Journal of Pervasive Computing and Communications.

[45] Arun, M., Baraneetharan, E., Kanchana, A. and Prabu, S., 2020. Detection and monitoring of the asymptotic COVID-19 patients using IoT devices and sensors. International Journal of Pervasive Computing and Communications.

[46] Rajendran, G.B., Kumarasamy, U.M., Zarro, C., Divakarachari, P.B. and Ullo, S.L., 2020. Land-use and land-cover classification using a human groupbased particle swarm optimization algorithm with an LSTM Classifier on hybrid pre-processing remote-sensing images. Remote Sensing, 12(24), p.4135.

[47] Parameshachari, B.D., Kiran, R.P., Rashmi, P., Supriya, M.C., Rajashekarappa and Panduranga, H.T., 2019, January. Controlled partial image encryption based on LSIC and chaotic map. In ICCSP (pp. 60-63).

[48] K. Yu, L. Tan, L. Lin, X. Cheng, Z. Yi and T. Sato, "Deep-Learning-Empowered Breast Cancer Auxiliary Diagnosis for 5GB Remote E-Health," IEEE Wireless Communications, vol. 28, no. 3, pp. 54-61, June 2021, doi: 10.1109/MWC.001.2000374.

[49] K. Yu, Z. Guo, Y. Shen, W. Wang, J. C. Lin, T. Sato, "Secure Artificial Intelligence of Things for Implicit Group Recommendations", IEEE Internet of Things Journal, 2021, doi: 10.1109/JIOT.2021.3079574.

[50] K. Yu, L. Tan, S. Mumtaz, S. Al-Rubaye, A. AlDulaimi, A. K. Bashir, F. A. Khan, "Securing Critical Infrastructures: Deep Learning-based Threat Detection in the IIoT", IEEE Communications Magazine, 2021.

[51] L. Tan, K. Yu, A. K. Bashir, X. Cheng, F. Ming, L. Zhao, X. Zhou, "Towards Real-time and Efficient Cardiovascular Monitoring for COVID-19 Patients by 5G-Enabled Wearable Medical Devices: A Deep Learning Approach", Neural Computing and Applications, 2021, https://doi.org/10.1007/s00521021-06219-9.

[52] L. Tan, K. Yu, F. Ming, X. Cheng, G. Srivastava, "Secure and Resilient Artificial Intelligence of Things: a HoneyNet Approach for Threat Detection and Situational Awareness", IEEE Consumer Electronics Magazine, 2021, doi: 10.1109/MCE.2021.3081874. 